\title{
Investigation of Influence of Adjustments in Cyclist Arm Position on Aerodynamic Drag Using Computational Fluid Dynamics ${ }^{\dagger}$
}

\author{
Knut Erik Teigen Giljarhus ${ }^{1, *}$, Daniel Årrestad Stave ${ }^{1}$ and Luca Oggiano ${ }^{2}$ \\ 1 Department of Mechanical and Structural Engineering and Materials Science, University of Stavanger, PB \\ 8600, 4036 Stavanger, Norway; daniel.stave@outlook.com \\ 2 Nabla Flow AS, 4050 Sola, Norway; loggiano@nablaflow.no \\ * Correspondence: knut.e.giljarhus@uis.no; Tel.: +47-99265474 \\ + Presented at the 13th conference of the International Sports Engineering Association, Online, \\ 22-26 June 2020.
}

Published: 15 June 2020

\begin{abstract}
In professional cycling, even small adjustments in position could mean that valuable seconds are gained over the course of a time-trial race. This study investigates the influence of arm position on the aerodynamic drag of a cyclist. Based on a 3D scanned model of a professional cyclist, 64 alternate positions are generated. The parameters that are investigated are the distance between elbows, elbow extension, and distance between hands. Computational fluid dynamics (CFD) simulations of all positions are performed, and a regression model is built from the results. The results indicate that the optimal posture is achieved for a minimum in all investigated parameters, which means that the hands and elbows should be kept together with hands up towards the face. Furthermore, elbow extension seems to be the most crucial parameter, followed by the distance between elbows, and then by the distance between the hands. The presented methodology can be applied to study other parameters relevant to cycling aerodynamics or be applied to other sport activities as well.
\end{abstract}

Keywords: cycling aerodynamics; cyclist posture; CFD; OpenFOAM

\section{Introduction}

The investigation of cycling aerodynamics has undergone considerable development in recent years. Grappe et al. [1] measured the total resisting force for a pedaling cyclist in different positions using a mechanism for measuring mechanical power. They reported that, at high speeds, approximately $90 \%$ of this force depends on the aerodynamic drag of air, and found a $27.8 \%$ reduction in drag area, $A C_{D}$, by changing the posture of the cyclist. The drag area is the product of the projected frontal area of the cyclist, and the drag coefficient is given by $A C_{D}=F_{D} / \frac{1}{2} \rho U^{2}$, where $F_{D}$ is the force in the cycling direction, $\rho$ is the air density, and $U$ is the velocity of the rider. Defraeye et al. [2] investigated the drag on a cyclist for three different positions using both wind tunnel experiments and computational fluid dynamics (CFD) modeling. They report agreement between the experimental and computational results with either instance of turbulence modeling, and state that "CFD is found to be a valuable tool to evaluate the drag of different cyclist positions and to investigate the influence of small adjustments in the cyclist's position." García-López et al. [3] studied the aerodynamic drag of five professional cyclists in four postures using wind tunnel experiments. They aimed to reduce the drag by modifying their posture and cycle equipment as well as the significance of these modifications, and reported a $14 \%$ reduction in drag by adjusting the posture alone. Beaumont et al. [4] investigated the aerodynamic effect of helmet shape and head positioning on cyclist drag using CFD, and reported the maximum difference of $6.4 \%$ by adjusting 
the head position alone and a difference of $1.5 \%$ by changing helmet shape for a fixed head position. Blocken et al. [5] analyzed the drag of a drafting cyclist, i.e., one cyclist riding behind another, in different postures using both CFD and wind tunnel experiments. They found that both the lead and trailing cyclists experience reduced drag from such a formation, with maximal drag reductions of $2.6 \%$ and $27.1 \%$, respectively, although not for the same posture. Based on these studies, it is evident that there is a potential gain to be obtained by looking further into the influence of posture on cycling aerodynamics. In this work, the effect of arm position is studied using CFD simulations. A 3D scanned model of a rider from the professional cycling team Team INEOS was used for the analysis. The base geometry was digitally modified to systematically investigate the effect of arm position on the aerodynamic drag. Three parameters were studied: The elbow extension, the shoulder abduction, and the elbow rotation. The extreme positions for each parameter are shown along with the base geometry in Figure 1. The boundaries of the modifications are defined in such a way that all the resulting postures satisfy the regulations by the International Cycling Union (UCI) [6].

It should be noted that by digitally modifying a posture, there is some uncertainty related to whether the modified position is realistic from an anatomic perspective. In addition, the position could be uncomfortable for the rider or lead to faster fatigue due to higher lactic acid accumulation. These aspects must be considered when evaluating the simulation results.
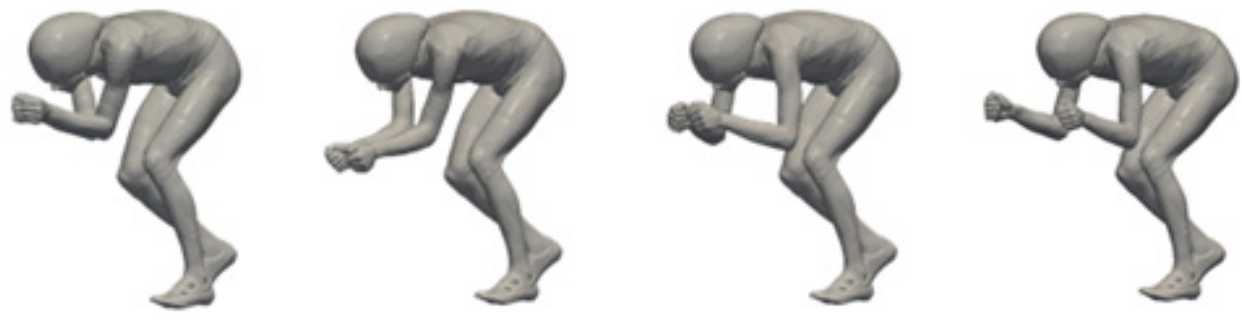

Figure 1. Illustration of the positional parameters considered. From left to right: Base position, maximum elbow extension, maximum shoulder abduction, and maximum elbow rotation and shoulder abduction.

\section{Materials and Methods}

The simulations were performed in the open-source simulation software OpenFOAM [7]. Quasisteady-state simulations were used with a Reynolds-Averaged Navier-Stokes (RANS) turbulence model; more specifically, the k- $\omega$ Shear Stress Transport (SST) turbulence model [8]. A recent study by Mannion et al. [9] demonstrated that this model gave good agreement with wind tunnel experiments. The size of the computational domain is $L \times W \times H=18 \times 12 \times 12 \mathrm{~m}$, giving a blockage ratio of $0.24 \%$. The cyclist is placed centrally in the domain, $6 \mathrm{~m}$ from the inlet. A non-pedaling cyclist model is used with the assumption that the flow past a static leg is similar to the flow past a cyclist's rotating leg at the appropriate phase of leg rotation. A study of Crouch et al. [10] showed minor variation between the instantaneous drag and primary vortical structures of a pedaling cyclist compared to a stationary cyclist with the pedals in the same position. The bicycle is also removed from the simulation to keep a reasonable simulation time, since the purpose of this study is to investigate the cyclist posture. For each of the three posture parameters considered, four variations are simulated. This gives a total of $4^{3}=64$ simulations.

A hex-dominated unstructured grid is applied with refinements near the cyclist body, as illustrated in Figure 2. The cell size for the coarsest level is $0.36 \mathrm{~m}$, and the total number of cells is around 7 million. Prism layers are inserted near the body to resolve the thin boundary layer near the cyclist. The average non-dimensional distance to the first grid cell is $y+\approx 2$ for the simulations presented here. For the given inlet velocity, this gives a distance to the first grid cell of approximately $20 \mu \mathrm{m}$. These grid parameters are similar to values reported in other studies, such as those of Blocken et al. [5] and Mannion et al. [7]. The expansion ratio from one layer to the next is set to 1.2. A closeup of the prism layer near the body is shown in the inset of Figure 2. 
The inlet boundary has a uniform constant velocity of $15 \mathrm{~m} / \mathrm{s}$, with zero gradient for pressure. Low turbulent levels are assumed with a turbulent intensity of $0.5 \%$. At the outlet, a fixed pressure is set with zero gradient for the remaining variables. The sides of the domain use a slip boundary condition. The cyclist body has a no-slip boundary condition with zero roughness and a blending wall function.

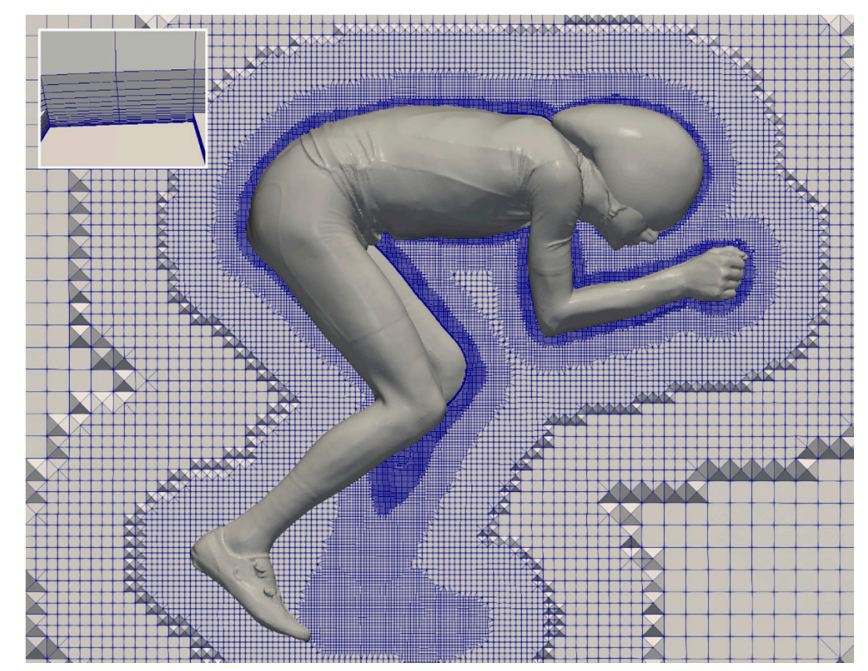

Figure 2. Illustration of grid refinement around the cyclist. The inset in the upper left shows a closeup of the prism layers near the cyclist body.

\section{Results and Discussion}

\subsection{Overview of Drag Forces and Regression Model}

The computed drag force for the reference position is $17.8 \mathrm{~N}$, which corresponds to a drag area of $A C_{\mathrm{d}}=0.148 \mathrm{~m}^{2}$. This is in line with other reported CFD results for the drag area for a cyclist; Defraye et al. [2] reported $A C_{\mathrm{d}}=0.142 \mathrm{~m}^{2}$, Blocken et al. [5] reported $A C_{\mathrm{d}}=0.135 \mathrm{~m}^{2}$, and Beaumont et al. [4] reported $A C_{\mathrm{d}}=0.138 \mathrm{~m}^{2}$. Of the total drag force, the friction drag only accounts for around $5 \%$, while the remaining $95 \%$ comes from the pressure drag.

Figure 3 presents the results in terms of drag force as a function of elbow extension and distance between hands (elbow rotation) for increasing distance between underarms (shoulder abduction). All distances are given relative to the maximum distances shown in Figure 1. A fourth-order regression model is also plotted along with the simulated results. Although care should be taken when interpreting the results from the regression model, it can still be useful in understanding trends of the simulation parameters. Overall, a minimum in all considered parameters gives the lowest drag forces. This means that the underarms should be kept as close as possible, the hands as close together as possible, and the elbow extension as small as possible. This means that none of the studied positions give a lower drag than the base position. The next sections discuss some other interesting observations that can be gleaned from the results.

\subsection{Influence of Elbow Extension}

Figure 4 shows a comparison between the surface pressure for the base position and for the position with maximum elbow extension. Regions with higher pressure on the torso, as well as regions with lower pressure on the upper arm and hip, can be observed for the position with high elbow extension. This is further illustrated in Figure 5, which shows the velocity magnitude in a slice normal to the flow direction, just behind the cyclist body. Since most of the total drag force is comprised of pressure drag, the size of the momentum deficit closely corresponds to the drag force. Since more of the air enters towards the torso and thighs when the lower arms are lowered, this 
causes a higher degree of separation when the flow passes over the hips. This results in a higher flow resistance for the cyclist.

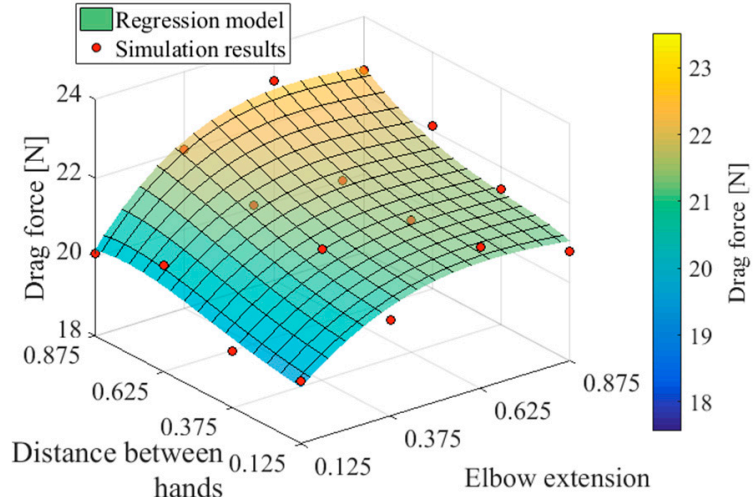

hands

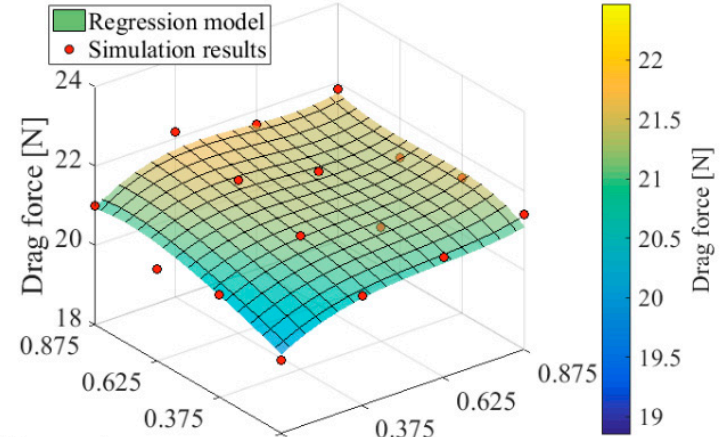

Distance between $0.125 \quad 0.125$ Elbow extension hands

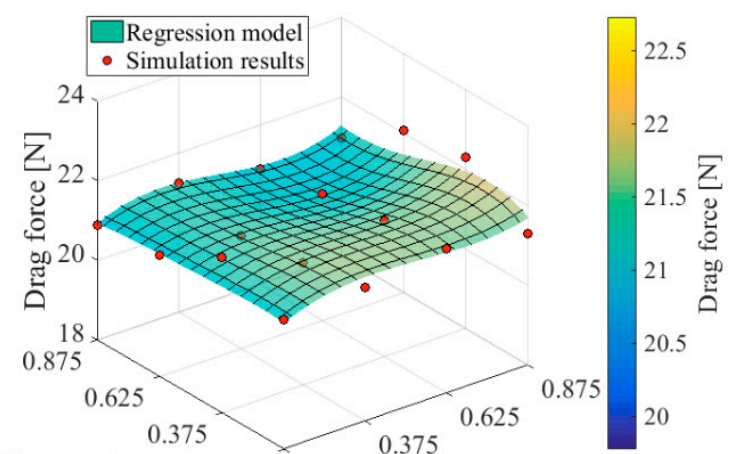

$\begin{gathered}\text { Distance between } 0.125 \\ \text { hands }\end{gathered}$
0.125 Elbow extension

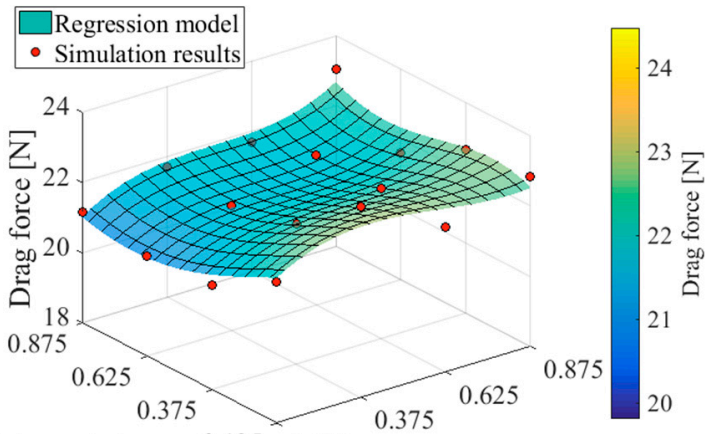

Distance between $0.125 \quad 0.125$ hands

Figure 3. Plots of simulated results and regression model. Top left: Low shoulder abduction, i.e., elbows close together. Top right: Medium shoulder abduction. Bottom left: Medium/high shoulder abduction, i.e., elbows shoulder-width apart. Bottom right: High shoulder abduction, i.e., elbows more than shoulder-width apart.
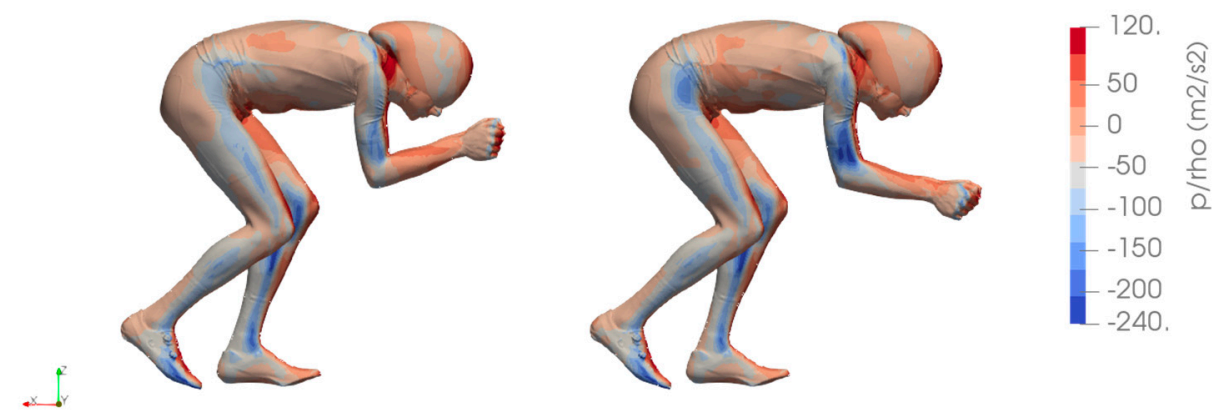

Figure 4. Comparison of relative pressure, $p / \rho\left[\mathrm{m}^{2} / \mathrm{s}^{2}\right]$, on cyclist body between base position and maximum elbow extension. 

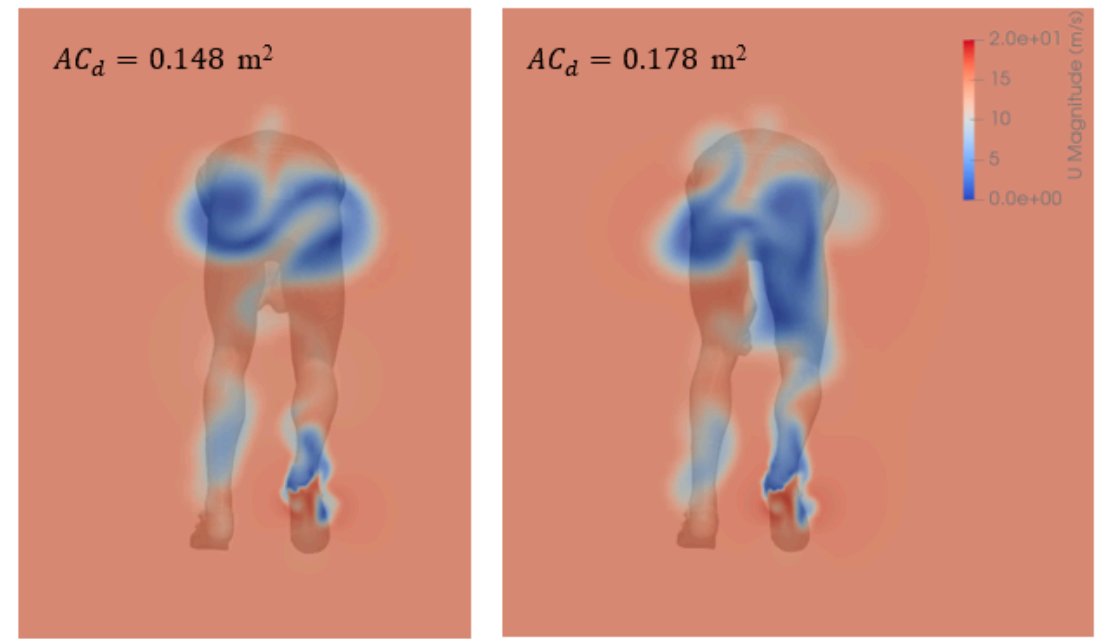

Figure 5. Slice plot of velocity magnitude right behind the cyclist body. Left: Base position. Right:

Maximum elbow extension.

\subsection{Influences of Shoulder Abduction and Elbow Rotation}

The influences of shoulder abduction and elbow rotation are best studied together. Figure 6 shows a horizontal slice plot of velocity magnitude comparing for the base position and a position with a separation distance between the underarms, i.e., medium shoulder abduction and medium elbow rotation. With separated arms, air is allowed to pass between the arms towards the torso and hips. Similarly to the position with higher elbow extension, this causes a larger wake behind the cyclist due to a higher degree of flow separation behind the right thigh. With arms kept together, the velocity is low in the region just beneath the torso, and the wake behind the body remains narrower than for separated arms.

Another interesting observation is the effect of keeping the hands together but separating the elbows, i.e., high shoulder abduction and low elbow rotation. This is a position adopted, for instance, by downhill skiers to minimize frontal area and give a streamlined position to reduce drag. However, for the cyclist, the effect is increased drag compared to the base position. This is because, for a downhill skier, the torso can be kept much closer to the legs, which does not allow air to enter and hit the torso, causing a larger wake. For a cyclist, the ideal position appears to be having the arms parallel and as close together as possible.

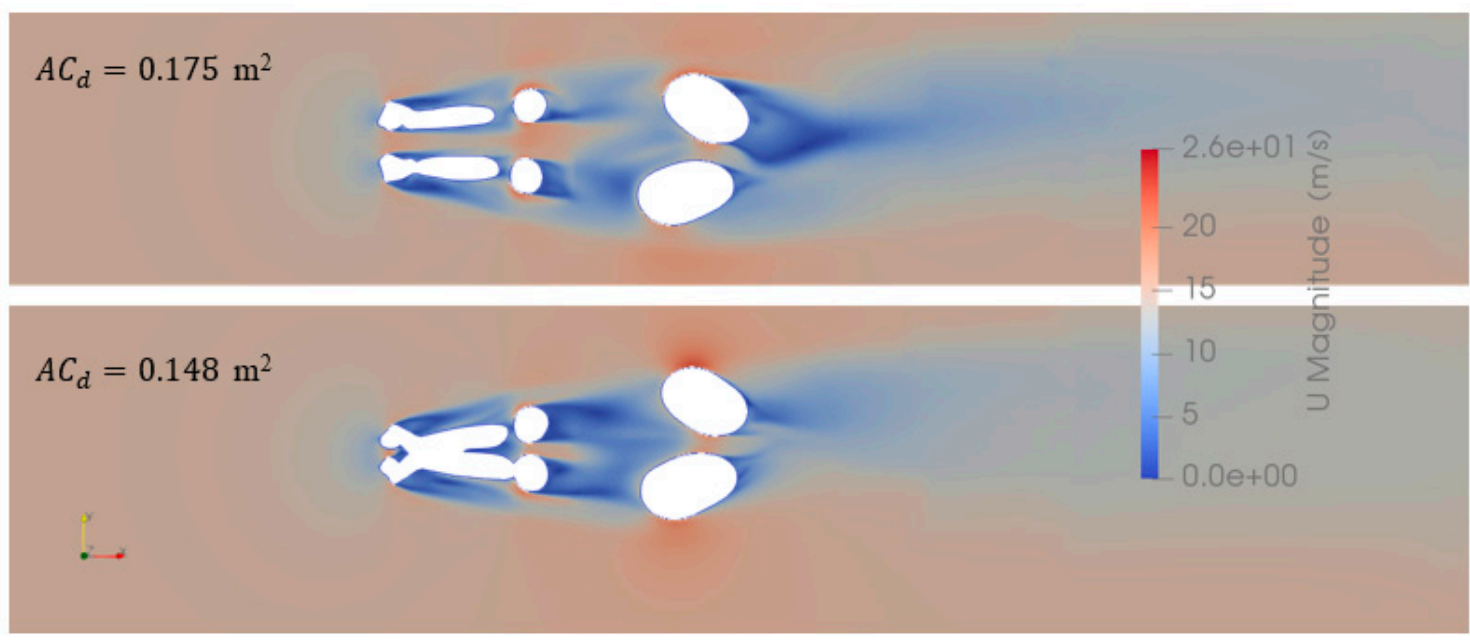

Figure 6. Horizontal slice plot of velocity magnitude comparing influences of underarm separation. Top: Medium shoulder abduction and elbow rotation. Bottom: Base position. 


\section{Conclusions}

This work presented a systematic evaluation of the influence of arm position in cycling aerodynamics. The influence of elbow extension, distance between hands, and distance between elbows was considered. Four values for each parameter were considered, resulting in a total of 64 geometry models. All of these were simulated using the OpenFOAM simulation software. The results show that the optimal position is to keep elbows and hands together and the elbow extension as small as possible. Increased elbow extension and keeping a distance between underarms lead to a larger wake due to the increased flow separation at the outside of the thighs.

Further work of this research is to validate the postures that, according to CFD, experienced the least drag force in either wind tunnel or time-trial experiments. In addition, further microoptimization of the position could potentially be obtained by investigating more points near the optimal position. For such a study, also including the handlebars in the study could be relevant. Another promising venue is to combine the simulations with an optimization algorithm instead of performing a parametric study. Additionally, only a single rider was considered in this study, and there could be significant individual differences in optimal positions when looking at small variations near the optimal point found in this study.

Author Contributions: KETG and LO conceived the idea for the work. KETG wrote the manuscript, performed simulations and did post-processing work. DÅS prepared the geometry models and performed simulations. All authors helped shape the research and analysis and contributed to the final manuscript.

Acknowledgments: The authors acknowledge the support of Scott Drawer and Carsten Jeppsen from Team Sky. The 3D scanned geometry model was provided by TotalSim Ltd. We also thank the anonymous reviewers for their constructive comments. The study was performed without any external funding.

Conflicts of Interest: The authors declare no conflict of interest.

\section{References}

1. Grappe, F.; Candau, R.; Belli, A.; Rouillon, J.D. Aerodynamic drag in field cycling with special reference to the Obree's position. Ergonomics 1997, 40, 1299-1311.

2. Defraeye, T.; Blocken, B.; Koninckx, E.; Hespel, P.; Carmeliet, J. Aerodynamic study of different cyclist positions: CFD analysis and full-scale wind-tunnel tests. J. Biomech. 2010, 43, 1262-1268.

3. García-López, J.; Rodríguez-Marroyo, J.A.; Juneau, C.E.; Peleteiro, J.; Martínez, A.C.; Villa, J.G. Reference values and improvement of aerodynamic drag in professional cyclists. J. Sports Sci. 2008, 26, 277-286.

4. Beaumont, F.; Taiar, R.; Polidori, G.; Trenchard, H.; Grappe, F. Aerodynamic study of time-trial helmets in cycling racing using CFD analysis. J. Biomech. 2018, 67, 1-8.

5. Blocken, B.; Defraeye, T.; Koninckx, E.; Carmeliet, J.; Hespel, P. CFD simulations of the aerodynamic drag of two drafting cyclists. Comput. Fluids 2013, 71, 435-445.

6. Internationale UC. Clarification Guide of the UCI Technical Regulation. In: Internationale UC, (ed.). 4/5/2020 ed.: Union Cycliste Internationale, 2020.

7. Weller, H.G.; Tabor, G.; Jasak, H.; Fureby, C. A tensorial approach to computational continuum mechanics using object-oriented techniques. Comput. Phys. 1998, 12, 620-631.

8. Menter, F.R.; Kuntz, M.; Langtry, R. Ten years of industrial experience with the SST turbulence model. In Proceedings of the fourth international symposium on turbulence, heat and mass transfer, Antalya, Turkey, 12-17 October 2003; pp. 625-632.

9. Mannion, P.; Toparlar, Y.; Blocken, B.; Hajdukiewicz, M.; Andrianne, T.; Clifford, E. Improving CFD prediction of drag on Paralympic tandem athletes: influence of grid resolution and turbulence model. Sports Eng. 2018, 21(2), 1-13.

10. Crouch, T.N.; Burton, D.; Brown, N.A.T.; Thompson, M.C.; Sheridan, J. Flow topology in the wake of a cyclist and its effect on aerodynamic drag. J. Fluid Mech. 2014, 748, 5-35. 